\title{
Effects of Thermal Pressing on ZnO Nanoparticle Layers Deposited by Drop Casting
}

\author{
Toshiyuki Yoshida, ${ }^{*}$ Kazato Shinohara, Daiki Itohara, and Yasuhisa Fujita \\ Interdisciplinary Graduate School of Science and Engineering, \\ Shimane University, 1060 Nishikawatsu, 690-8504 Matsue, Japan \\ (Received 23 March 2016; Accepted 19 April 2016; Published 28 May 2016)
}

\begin{abstract}
$\mathrm{ZnO}$ nanoparticle (NP) layers were formed using a simple drop casting process. Applying thermal pressing clearly improved the surface morphology and electrical properties of the as-deposited ZnO NP layers as well as significantly enhanced the transistor characteristics of the NP layers. The effects of thermal pressing on the film surface properties were evaluated by X-ray photoelectron spectroscopy, Kelvin probe microscopy, and photoluminescence spectroscopy. Results suggest that thermal pressing improved the transistor characteristics by decreasing NP surface defects such as oxygen vacancies. [DOI: 10.1380/ejssnt.2016.175]
\end{abstract}

Keywords: X-ray photoelectron spectroscopy; Zinc oxide; Powders; Semiconducting films; Transistor

\section{INTRODUCTION}

Nanoparticle (NP) semiconductor films have attracted considerable attention as channel layers for thin-film transistors (TFTs) because of their extremely low-cost fabrication processes. Another attractive feature is their adaptability to flexible substrates exhibiting various surface morphologies such as curved and non-atomically flattened surfaces. The surfaces of oxide semiconductors such as $\mathrm{ZnO}$ do not form an insulating layer when dispersed in water. Therefore, many efforts have been made to incorporate ZnO NP layers into TFTs. [1-7] Generally, NP layers have been generated mainly by annealing and plasma treatments to control surface chemistry and crystallinity. However, few reports have addressed the degree of adhesion and mutual binding between individual NPs. To enhance binding and minimize gaps between NPs, thermal pressing was applied to drop-cast ZnO NP layers formed on quartz substrates, which is expected to improve the intrinsic properties of particle-based films prior to conventional surface treatments such as annealing and plasma treatments. The resulting TFTs were implemented in metal-semiconductor field-effect transistor (MESFET) structures.

\section{EXPERIMENTAL}

$\mathrm{ZnO}$ NPs were synthesized by arc-discharge-mediated gas evaporation [8] using dry air at 610 Torr and an arc current of $30 \mathrm{~A}$. The obtained NPs $(0.1 \mathrm{~g})$ were dispersed in water $(20 \mathrm{~g})$ by ultrasonic homogenization $(150 \mathrm{~W}, 3$ min). After centrifugation (2000 g, 1 min), a ZnO NP dispersion with a median particle size of $250 \mathrm{~nm}$ was obtained. The dispersion was deposited dropwise using a micropipette $(5 \mu \mathrm{l} /$ drop $) 100$ times on quartz substrates heated at $500{ }^{\circ} \mathrm{C}$ (Fig. 1(a)). A relatively high temperature $\left(500{ }^{\circ} \mathrm{C}\right)$ was used to minimize the evaporation time and improve cohesion of particles. Although the sudden boiling could also potentially enhance the gap between NPs, this method provided samples suitable for verifying the effects of the following thermal pressing. Finally, layers were pressed at pressures of $300-900 \mathrm{~kg} / \mathrm{cm}^{2}$ at $300^{\circ} \mathrm{C}$

\footnotetext{
* Corresponding author: yosisi@ecs.shimane-u.ac.jp
}

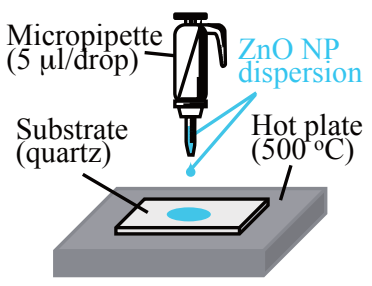

(a)

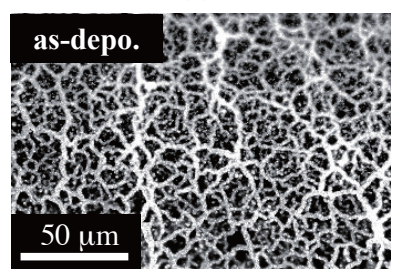

(c)

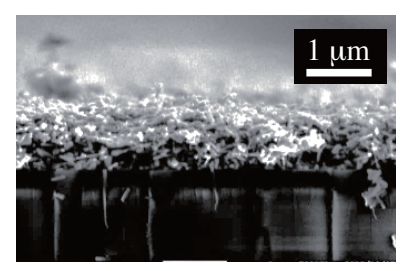

(b)

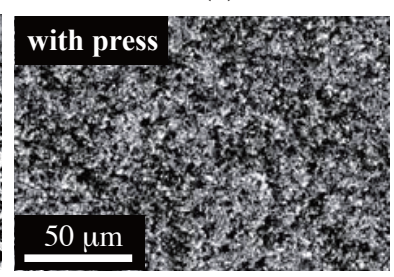

(d)
FIG. 1. (a) Schematic of the drop casting method. (b) Crosssectional FESEM image of drop-cast film. Optical microscopy images of film surfaces (c) before and (d) after thermal pressing.

for $30 \mathrm{~s}$. The film morphologies were examined by optical (AxioImage-2, Zeiss) and field-emission scanning electron microscopes (FESEM; JSM-7001FA, JEOL, $5 \mathrm{kV}$ ). Elemental analyses were performed by X-ray photoelectron spectroscopy (XPS) with $\mathrm{Al} \mathrm{K} \alpha$ radiation. Binding energies were calibrated by shifting the $\mathrm{C} 1 \mathrm{~s}$ peak position to $285.0 \mathrm{eV}$. [9-12] The sheet resistance was determined independently by the transfer length method for an electrode distance of 100-500 $\mu \mathrm{m}$. Electron mobility was evaluated by the van der Pauw method. Surface potentials were analyzed by Kelvin force microscopy (KFM; SKP5050, KP Technology) and optical properties were measured by photoluminescence spectroscopy (PL; FluoroMax-4, Horiba) using monochromatic excitation at $325 \mathrm{~nm}$.

\section{RESULTS AND DISCUSSION}

Figure 1(b) shows a cross-sectional FESEM image of the as-deposited ZnO NP layer. The substrate surface was covered with an NP layer with an estimated thickness of about $1.0 \mu \mathrm{m}$, which agreed with the value calculated based on the drop-cast area and the total volume of 


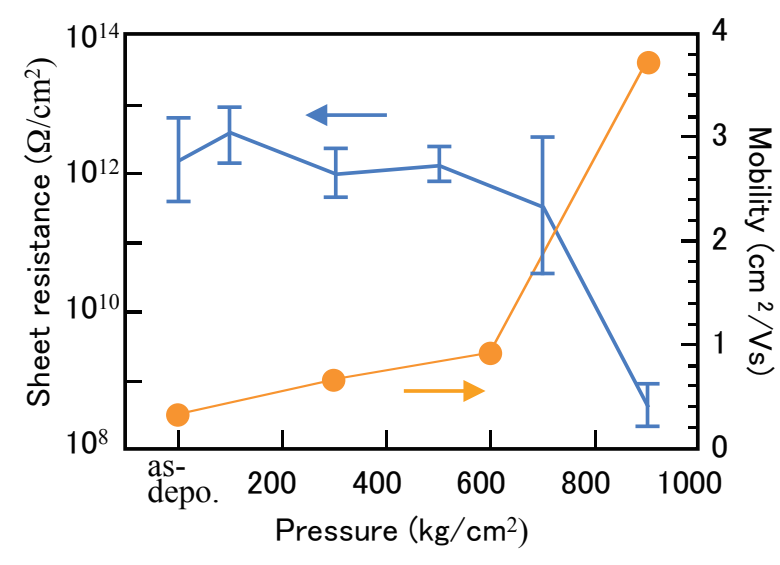

FIG. 2. Sheet resistance and mobility as functions of pressure during thermal pressing. Data obtained before pressing are denoted "as-depo".

the deposited particles. Figures 1(c) and 1(d) show optical microscopy surface images before and after thermal pressing $\left(900 \mathrm{~kg} / \mathrm{cm}^{2}\right.$ and $\left.300{ }^{\circ} \mathrm{C}\right)$, respectively. The asdeposited surface displayed a mesh-like morphology, indicating that individual NPs were relatively spread out in the film. The thermal pressing treatment decreased the average separation between NPs, leading to a tighter surface morphology.

Figure 2 shows the influence of pressure on sheet resistance and electron mobility during thermal pressing. Before thermal pressing, the films exhibited extremely high sheet resistance and low mobility. A small improvement was observed when pressed below $600 \mathrm{~kg} / \mathrm{cm}^{2}$. However, when pressing at $900 \mathrm{~kg} / \mathrm{cm}^{2}$, the sheet resistance dropped and mobility increased drastically.

Figures 3(a)-3(d) show XPS measurements of the $\mathrm{O} 1 \mathrm{~s}$ and $\mathrm{Zn} 2 p_{3 / 2}$ signals, acquired an escape angle of $45^{\circ}$ before and after thermal pressing at $900 \mathrm{~kg} / \mathrm{cm}^{2}$ and 300 ${ }^{\circ} \mathrm{C}$. The $\mathrm{O} 1 \mathrm{~s}$ spectrum (Fig. 3(a)) consisted of two peaks at binding energies of 530.3 and $531.8 \mathrm{eV}$, which were assigned to typical wurtzite $\mathrm{ZnO}$ and $\mathrm{OH}$ groups, respectively. [13-18] The inset shows the $\mathrm{OH}$ group ratios in the $\mathrm{O} 1 \mathrm{~s}$ spectrum at escape angles of $45^{\circ}$ and $15^{\circ}$. The quasi-similarity of these ratios indicates that this functional group was evenly distributed in the NP layer. The Zn $2 p_{3 / 2}$ spectrum was consistent with typical wurtzite $\mathrm{ZnO}$ (Fig. 3(b)) and is in agreement with previous reports. [13, 15, 17-19]

The proportions of chemical components including the $\mathrm{OH}$ group ratios did not change after thermal pressing (Figs. 3(c) and 3(d)); however, the $\mathrm{O} 1 s(\mathrm{ZnO})$ and $\mathrm{Zn}$ $2 p_{3 / 2}$ peak positions shifted to higher energies by $0.15 \mathrm{eV}$. Valence band spectra before and after thermal pressing $\left(900 \mathrm{~kg} / \mathrm{cm}^{2}\right.$ and $\left.300{ }^{\circ} \mathrm{C}\right)$ are shown in Fig. 3(e). The peak heights were normalized with respect to the $\mathrm{O} 2 p$ $\pi$ signals. Before thermal pressing, the valence band full spectrum (inset, Fig. 3(e)) displayed clear peaks for $\mathrm{ZnO}$ binding orbitals, consistent with those previously reported for single-crystal surfaces. [20, 21] The difference between the Fermi level and the valence band edge increased by about $0.15-0.17 \mathrm{eV}$ after thermal pressing.

KFM profiles were also obtained by studying the $\mathrm{ZnO}$
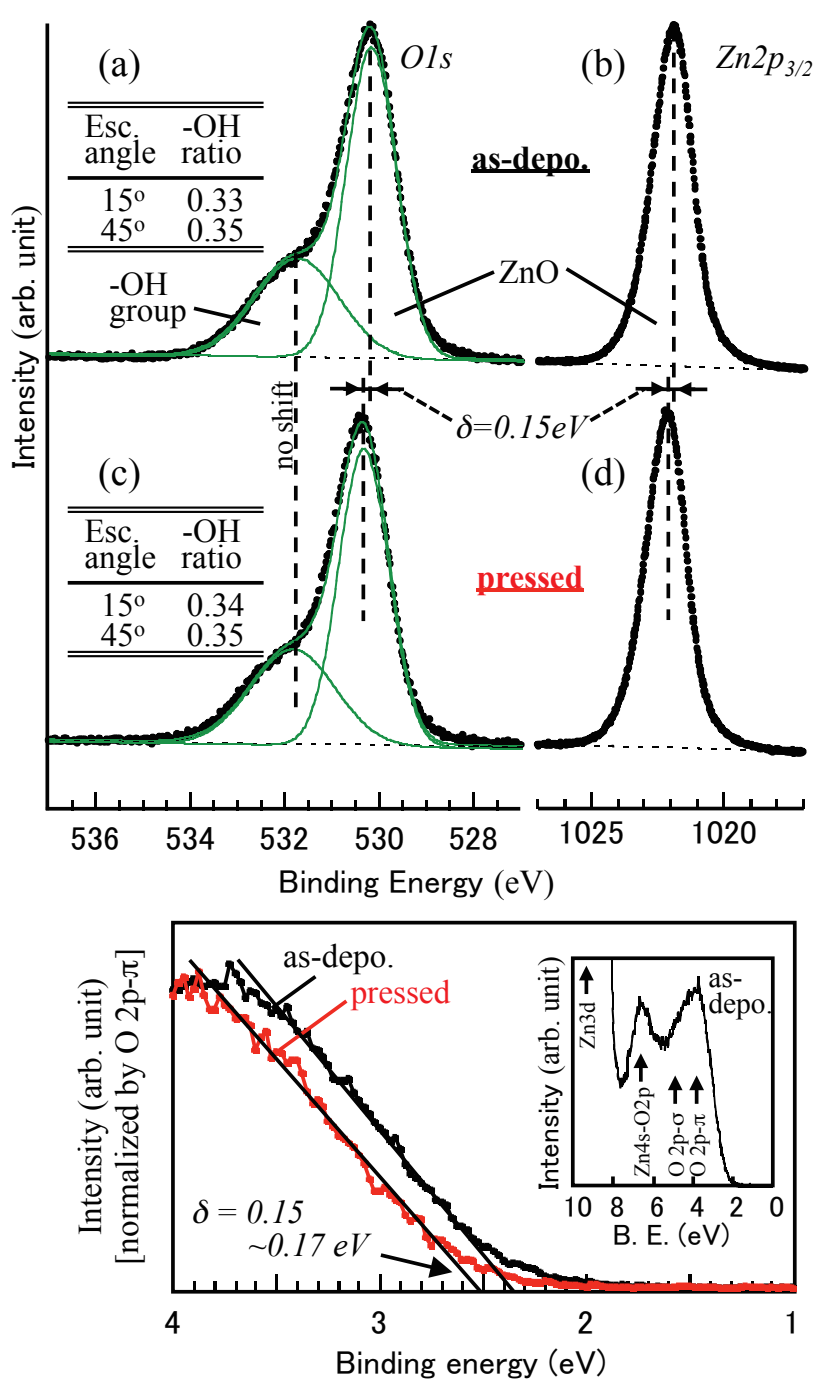

(e)

FIG. 3. XPS of O $1 s$ and Zn $2 p_{3 / 2}(\mathrm{a}, \mathrm{b})$ before and $(\mathrm{c}, \mathrm{d})$ after thermal pressing. The escape angle was $45^{\circ}$. Inset: integrated intensity ratios are shown for $\mathrm{OH}$ groups in the O1s spectra. (e) XPS valence band spectra for as-deposited and thermally pressed $\mathrm{ZnO} \mathrm{NP}$ layers.

NP layer between the $\mathrm{Au}$ and $\mathrm{Al}$ regions to facilitate calibration (Fig. 4). Black solid and red dashed lines correspond to as-deposited and pressed samples $\left(900 \mathrm{~kg} / \mathrm{cm}^{2}\right.$, $300{ }^{\circ} \mathrm{C}$ ), respectively. Unfortunately, the profile of the pressed sample presented a non-uniform curve in the $\mathrm{ZnO}$ NP region. Drop casting may have caused variations in the NP layer thickness, resulting in an uneven pressure distribution during thermal pressing. The pressed portions of the films showed a $157 \mathrm{meV}$ work function decrease. This decreased work function corresponds to an increase in the energy difference between the Fermi level and the valence band edge, consistent with the XPS results. This confirms that thermal pressing caused relaxation of the surface band bending.

Figures 3(a) and 3(c) show that the $\mathrm{OH}$ signal positions did not change during thermal pressing. These groups behaved independently from the $\mathrm{ZnO}$ signals and escape angle, indicating a separate environment of these groups from the $\mathrm{ZnO}$ NPs. The $\mathrm{OH}$ groups are likely distributed 


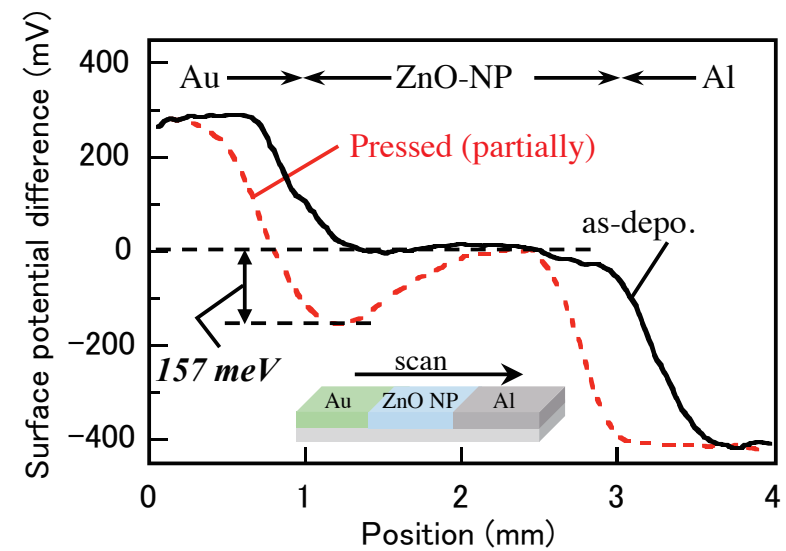

FIG. 4. KFM surface potentials for $\mathrm{Au} / \mathrm{ZnO}-\mathrm{NP} / \mathrm{Al}$ structures before and after thermal pressing.

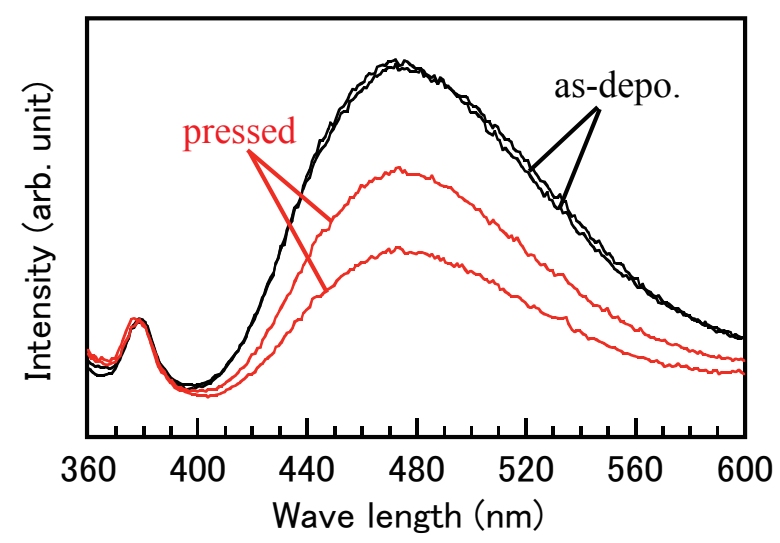

FIG. 5. PL spectra of as-deposited and thermally pressed $\mathrm{ZnO}$ $\mathrm{NP}$ layers. Intensities of $\mathrm{ZnO}$ exciton signals are adjusted for comparison.

on the surface of each NP within the layer.

Figure 5 shows PL spectra of $\mathrm{ZnO} \mathrm{NP}$ layers in two asdeposited and thermally pressed samples at $900 \mathrm{~kg} / \mathrm{cm}^{2}$ and $300{ }^{\circ} \mathrm{C}$, with the intensities adjusted for comparison. All spectra displayed clear $\mathrm{ZnO}$ exciton peaks, and broad peaks ranging from 400 to $600 \mathrm{~nm}$ were assigned to various defects such as oxygen vacancies. [22] The peak at approximately $500 \mathrm{~nm}$ decreased for core-shell structured NPs having a silica shell (data not shown), which indicates passivation of surface defects. Furthermore, the PL spectrum obtained from binder-coated ZnO NPs (Fig. 3 in Ref. [8], NPs with surface defect passivation) showed a signal reduction in the same range. NP formation conditions in Ref. [8] were not exactly similar to those used in this study but exploited an identical gas evaporation method. Therefore, the signal observed between 400 and $600 \mathrm{~nm}$ in Fig. 5 are also likely to originate from NP surface defects. In this study, signals assigned to defects clearly decreased after thermal pressing. Compared with XPS measurements, PL measurements were more representative of the bulk sample. Therefore, the defect reduction phenomenon occurred mainly inside the NP layer. The reduction in the proportion of NP surfaces in the layers may explain the improvement induced by thermal

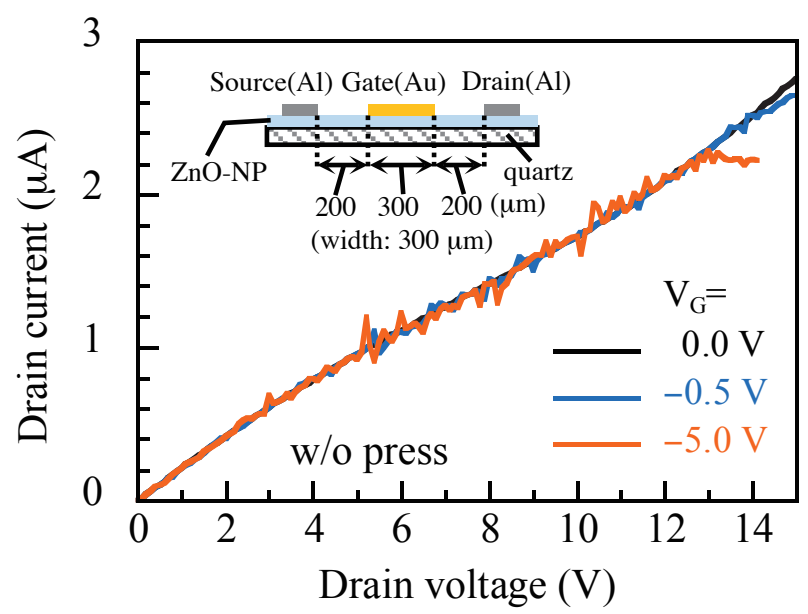

(a)

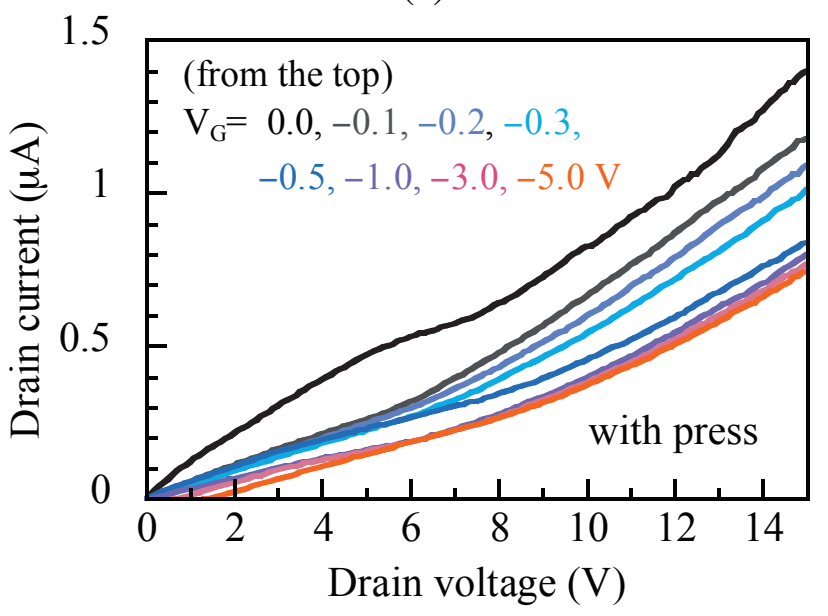

(b)

FIG. 6. $I_{D}-V_{D}$ characteristics of MESFETs on (a) asdeposited and (b) thermally pressed $\mathrm{ZnO}$ NP layers.

pressing. In this case, oxygen vacancies at NP surfaces were compensated by $\mathrm{OH}$ oxygen atoms at neighboring $\mathrm{NP}$ surfaces by heating at high pressure. However, because oxygen vacancies near the surface of the NP layer were not in contact with other NPs, only a few defects inside the NP layer were compensated. Generally, surface potential is sensitive to variations of defect density, and in this study, the defect density resulted in relaxation of band bending by $0.15 \mathrm{eV}$. This change was not detected by XPS (Fig. 3(a-d)) because these measurements are limited to probing the surface of samples. Difference among the rates of decrease in defects in the pressed samples shown in Fig. 5 may be explained by a reasoning similar to that which caused the non-uniform behavior shown in Fig. 4.

Finally, the as-deposited and thermally pressed $\mathrm{ZnO}$ NP layers were applied in TFTs. Current-voltage $\left(I_{D^{-}}\right.$ $\left.V_{D}\right)$ characteristics at the drain are shown in Figs. 4(a) and 4(b). A cross-sectional illustration of the TFTs is shown in the inset. $\mathrm{Al}$ and $\mathrm{Au}$ electrodes acted as ohmic source/drain electrodes and Schottky gate electrodes, respectively. Devices incorporating as-deposited NP layers showed poor transistor characteristics (Fig. 6(a)). A small 
variation in drain current with decreasing $V_{G}$ was only observed at $V_{D}$ values exceeding $13 \mathrm{~V}$. Conversely, the variability of drain current in response to gate voltage improved considerably for devices containing ZnO NP layers pressed at $900 \mathrm{~kg} / \mathrm{cm}^{2}$ and $300{ }^{\circ} \mathrm{C}($ Fig. 6(b)). Drain current dropped progressively with decreasing gate voltage, consistent with an n-type MESFET. This suggests thermal pressing induced a lower NP surface state defect density and caused relaxation of the NP layer surface band bending to produce a well-behaved metal-semiconductor interface. Moreover, the decrease in NP surface defects within the film introduced field effects deep into the NP layer. Here, transistor behavior was realized using $\mathrm{ZnO}$ NP layers formed by combining a simple drop casting method with thermal pressing.

\section{CONCLUSION}

In conclusion, $\mathrm{ZnO} \mathrm{NP}$ layers were formed on quartz substrates using a simple drop casting method. The ef- fects of an additional thermal pressing step were evaluated. The layers were incorporated into devices exhibiting MESFET behavior. Thermal pressing reduced NP surface defects and enhanced electrical properties of the layer by lowering sheet resistance and increasing electron mobility. Thermally pressed ZnO NP layers showed considerably improved transistor performance.

\section{ACKNOWLEDGMENTS}

We thank the Center for Integrated Research in Science, Shimane University, for facilitating the FESEM experiments through the Tatara Project (Ministry of Education, Culture, Sports, Science and Technology of Japan). We also thank the Research Center for Integrated Quantum Electronics (RCIQE), Hokkaido University, for facilitating the XPS experiments. This work was supported by the Japan Society for the Promotion of Science KAKENHI Grant Number 25870448.
[1] M. S. Rajachidambaram, T. Varga, L. Kovarik, R. Sanghavi, V. Shutthanandan, S. Thevuthasan, S.-Y. Han, C.H. Chang, and G. S. Herman, J. Vac. Sci. Technol. B 30, 041805 (2012).

[2] S. Walther, S. Polster, M. P. M. Jank, H. Thiem, H. Ryssel, and L. Frey, Adv. Power Technol. 22, 253 (2011).

[3] H. Faber, M. Klaumunzer, M. Voigt, D. Galli, B. F. Vieweg, W. Peukert, E. Spiecker, and M. Halik, Nanoscale 3, 897 (2011).

[4] H.-C. Huang and T.-E. Hsieh, Nanotechnology 21, 295707 (2010).

[5] N. Mechau, S. Bubel, D. Nikolova, and H. Hahn, Phys. Status Solidi A 207, 1684 (2010).

[6] S. Bubel, D. Nikolova, N. Mechau, and H. Hahn, J. Appl. Phys. 105, 064514 (2009).

[7] S. Lee, Y. Jeong, S. Jeong, J. Lee, M. Jeon, and J. Moon, Superlattices Microstruct. 44, 761 (2008).

[8] Y. Fujita, K. Moriyama, Y. Hiragino, Y. Furubayashi, H. Hashimoto, and T. Yoshida, Phys. Status Solidi C 11, 1260 (2014).

[9] V. I. Nefedov, Y. V. Salyn, G. Leonhardt, and R. Scheibe, J. Electron Spectrosc. Relat. Phenom. 10, 121 (1977).

[10] J. Haber, J. Stoch, and L. Ungier, J. Electron Spectrosc. Relat. Phenom. 9, 459 (1976).

[11] P. Zhang, C. Kong, W. Li, G. Qin, Q. Xu, H. Zhang, H. Ruan, Y. Cui, and L. Fang, Appl. Surf. Sci. 327, 154 (2015).
[12] O. Lupan, G. A. Emelchenko, V. V. Ursaki, G. Chai, A. N. Redkin, A. N. Gruzintsev, I. M. Tiginyanu, L. Chow, L. K. Ono, B. R. Cuenya, H. Heinrich, and E. E. Yakimov, Mater. Res. Bull. 45, 1026 (2010).

[13] J. Singh, A. Mukherjee, S. K. Sengupta, J. Im, G. W. Peterson, and J. E. Whitten, Appl. Surf. Sci. 258, 5778 (2012).

[14] L. Avalle, E. Santos, E. Levia, and V. A. Macagno, Thin Solid Films 219, 7 (1992).

[15] J. Liqiang, W. Dejun, W. Baiqi, L. Shudan, X. Baifu, F. Honggang, and S. Jiazhong, J. Mol. Catal. A: Chem. 244, 193 (2006).

[16] L.-J. Meng, C. P. Moreira de Sá, and M. P. dos Santos, Appl. Surf. Sci. 78, 57 (1994).

[17] Z. Wang, Y. Yue, and Y. Cao, Superlattices Microstruct. 65, 7 (2014).

[18] N. H. Erdogan, K. Kara, H. Ozdamar, H. Kavak, R. Esen, and H. Karaagac, J. Alloys Compd. 509, 8922 (2011).

[19] C. D. Wagner, W. M. Riggs, L. E. Davis, J. F. Moulder, and G. E. Muilenberg, in: Handbook of X-Ray Photoelectron Spectroscopy (Perkin-Elmer, Minnesota, 1979), p. 84.

[20] Z. Sojka and K. Klier, J. Electron Spectrosc. Relat. Phenom. 60, 155 (1992).

[21] V. G. Devyatov, A. E. Cherkashin, and S. V. Koshcheev, Nucl. Instrum. Methods Phys. Res. A 308, 225 (1991).

[22] S. J. Pearton, D. P. Norton, K. Ip, Y. W. Heo, and T. Steiner, Prog. Mater. Sci. 50, 293 (2005). 